\title{
A Obesidade Realmente Fortalece os Ossos?
}

A OBESIDADE E A OSTEOPOROSE SÃO duas doenças crônicas de alta prevalência na população geral no mundo moderno. Constituem um sério problema de saúde pública, pelo caráter assintomático ao longo de anos, porém com alta probabilidade de complicações como eventos cardiovasculares, diabetes tipo 2 e fraturas (1). A epidemia da obesidade se caracteriza pelo aumento progressivo da doença nos últimos 20 anos em todo o mundo, particularmente nos Estados Unidos, onde atinge cifras de $35 \%$ em adultos e $15 \%$ em crianças e adolescentes (1). A epidemia da osteoporose não poupa etnia ou ambiente e associa-se a alguns fatores como, por exemplo, envelhecimento da população pelo aumento da expectativa de vida e deficiência de vitamina D em escala global (2).

A associação entre obesidade e aumento da massa óssea é conhecida já de algum tempo. Em um estudo realizado em gêmeos dizigóticos e monozigóticos do sexo feminino, na vida adulta e na sua maioria no período pós-menopausa, utilizando a densitometria mineral óssea (DMO) areal pelo sistema de absorção de dupla energia de Rx (DXA), um grupo australiano demonstrou que a massa óssea e a massa adiposa estão sob forte regulação genética. A associação dos dois sofreu uma grande influência ambiental (3). Isto pode significar que fatores ambientais atuam na mesma direção tanto na massa óssea quanto na massa adiposa.

Neste número dos ABE\&M, da Silva e colaboradores publicam os resultados de um estudo retrospectivo relacionando densidade mineral óssea a obesidade (4). Foram avaliadas 588 mulheres com idades entre 41 e 60 anos (média de 54) que apresentavam menopausa por um período de l a 10 anos (média de 5), atendidas em uma clínica de densitometria óssea. Os critérios de exclusão foram: índice de massa corpórea (IMC) acima de $40 \mathrm{~kg} / \mathrm{m}^{2}$, história de doenças crônicas como insuficiência renal, hepática, cardíaca, doenças reumáticas e o uso de drogas como corticóides, antireabsortivos, fumo acima de 10 cigarros por dia e álcool em quantidades não relatadas. A DMO areal foi realizada pelo sistema DXA. Eles encontraram uma maior prevalência de osteoporose, na coluna lombar e no colo do fêmur, nas mulheres com peso normal quando comparadas àquelas com obesidade, assim como correlações positivas entre a DMO e IMC, e negativas entre $\mathrm{DMO}$ e idade e DMO e tempo de menopausa.

Vários mecanismos têm sido propostos para explicar o possível efeito benéfico da obesidade na massa óssea, tais como o aumento da carga mecânica sobre o esqueleto e fatores metabólicos (5). Além da adaptação do esqueleto ao aumento da força mecânica induzida pelo maior peso corporal, uma maior produção de estrógenos pelos adipócitos com conseqüente redução da remodelação óssea, além da resistência a insulina e hiperinsulinemia freqüentemente presentes em indivíduos obesos. A insulina pode ter efeito direto na formação óssea, como também pode induzir maior produção ovariana de esteróides sexuais e uma menor produção hepática da proteína que se liga aos hormônios sexuais (SHBG), levando a uma maior concentração sanguínea de estrógenos e andrógenos.

\section{editorial}

\section{FRANCISCO BANDEIRA}

Professor Adjunto de Endocrinologia e Epidemiologia, Faculdade de Ciências Médicas da Universidade de Pernambuco (UPE) e Chefe do Serviço de Endocrinologia e Diabetes, Hospital Agamenon Magalhães, SUS / UPE, Recife, PE. 
$\mathrm{Na}$ infância e adolescência, a obesidade pode levar a uma maior aceleração da maturação esquelética. Em um estudo comparativo realizado na Filadélfia entre 132 indivíduos no grupo etário de 4 a 20 anos com peso normal (IMC $<85$ percentil), e 103 obesos (IMC $\geq 95$ percentil), a obesidade se associou a uma maior maturação óssea como também a uma maior estatura para a idade (6). Os indivíduos foram classificados de acordo com o estágio puberal pelos critérios de Tanner e a DMO areal foi determinada pelo sistema DXA, pelo qual parâmetros como área do segmento esquelético $\left(\mathrm{cm}^{2}\right)$, conteúdo mineral ósseo $(\mathrm{g})$ e a DMO $\left(\mathrm{g} / \mathrm{cm}^{2}\right)$ puderam ser quantificados. Considerando-se todo o esqueleto, como também sítios específicos como coluna lombar, houve aumento significativo dos parâmetros analisados nos indivíduos obesos quando comparados àqueles com peso normal. Estas diferenças permaneceram significativas quando ajustadas para o grau de desenvolvimento puberal e sexo. Embora a obesidade infantil associe-se a um maior risco de fraturas de antebraço por quedas, em conseqüência de uma maior força de trauma, esses dados sugerem que a resistência óssea pode ser fortalecida pela obesidade.

Os hormônios envolvidos no controle do apetite têm importante papel no desenvolvimento puberal e na maturação esquelética, e a leptina, na maioria dos estudos, tem-se mostrado exercer um efeito favorável na formação óssea $(7,8)$, embora seus efeitos centrais e periféricos possam diferir, pois quando administrada diretamente nos ventrículos cerebrais de camundongos deficientes de leptina, pode causar perda óssea (5).

A resistência óssea ou qualidade óssea é quem determina a predisposição a fraturas de certa forma independente da DMO. De acordo com os critérios diagnósticos de osteoporose pela OMS, os indivíduos na faixa considerada intermediária, ou seja, osteopenia (escore t na DMO entre - 1 e -2,5), apresentam um risco substancial de desenvolverem fraturas vertebrais $\mathrm{e}$ não vertebrais, e 40 a $50 \%$ das fraturas vertebrais ocorrem ainda na fase de osteopenia (2).

Alguns estudos mais recentes têm mostrado que a associação obesidade e DMO pode não refletir a possível proteção da adiposidade contra a osteoporose. Tang e colaboradores (9) identificaram, em 4.126 indivíduos caucasianos, regiões genômicas compartilhadas para a determinação da massa adiposa e da DMO. Os genes em comum mais freqüentemente encontrados foram GDF8, TNF $\alpha$, Il6 e PONl (9).

Em um estudo realizado em dois grupos de indivíduos, caucasianos e chineses, as associações DMO e massa adiposa foram avaliadas (5). A população consistiu de 1.988 chineses, dos quais $42 \%$ eram mulheres na menopausa com média de idade de 27 anos, e de 4.489 caucasianos ( $45 \%$ mulheres) com média de idade de 48 anos. Embora tenha havido correlação positiva entre IMC e DMO, como também entre massa magra e DMO, houve correlações inversas entre DMO e a massa adiposa em quilograma e a DMO e o percentual de massa adiposa, as quais persistiram quando ajustadas para o peso corporal.

$\mathrm{Na}$ coorte Women's Health Initiative Observational Study, em observação nos Estados Unidos, o risco de fraturas clínicas foi avaliado em $\mathbf{5 . 2 8 5}$ mulheres diabéticas e 88.120 não diabéticas, com média de idade de 64 anos, durante um seguimento de 7 anos (10). Embora os dois grupos apresentassem média de ingestão de cálcio e vitamina $\mathrm{D}$ semelhantes (média de 1.043 e $1.185 \mathrm{mg} / \mathrm{dia}$, e 381 e 414 UI/dia) e uso de terapia de reposição estrogênica (31 e $45 \%$ ), o risco relativo de fratura foi de $29 \%$ para qualquer sítio, $41 \%$ para colo do fêmur e bacia e $28 \%$ para coluna, mesmo quando ajustados para a DMO. De fato, as pacientes diabéticas tinham DMO significativamente mais elevada tanto na avaliação basal como no seguimento anual. A 25-hidroxivitamina-D sanguínea não foi determinada.

A deficiência de vitamina D é encontrada freqüentemente em indivíduos obesos, e não apenas se associa ao excesso de adiposidade como também à deterioração do metabolismo dos carboidratos, como evidenciado na coorte britânica dos indivíduos nascidos em 1958 e avaliados aos 45 anos de idade (11).

As inter-relações entre obesidade e osteoporose estão longe de ser definidas cientificamente e ainda não totalmente conhecidas tanto quanto os mecanismos que levam às duas doenças. Ao contrário do conhecido efeito positivo do excesso ponderal na DMO, essas inter-relações parecem ser mais complexas, e isto pode vir a ser mais um exemplo de que DMO elevada nem sempre significa ossos mais fortes.

\section{REFERÊNCIAS}

1. Hill JO. Understanding and addressing the epidemic of obesity: An energy balance perspective. Endocr Rev 2006;27:750-61.

2. Bandeira F, Freese E. Prevalência de osteoporose e fraturas vertebrais em mulheres na pós-menopausa atendidas em serviços de referência. Rev Bras Epidemiol 2007;10:86-98.

3. Nguyen TV, Howard GM, Kelly PJ, Eisman JA. Bone mass, lean mass, and fat mass: same genes or same enviroments? Am J Epidemiol 1998;147:3-16.

4. Da Silva HG, Mendonça L, Conceição FL, Zahar SEV, Farias ML. Influence of obesity on bone density in postmenopausal women. Arq Bras Endocrinol Metab 2007;51/6:943-949.

5. Zhao LJ, Liu YJ, Liu PY, Hamilton J, Recker RR, Deng HW. Relationship of obesity with osteoporosis. J Clin Endocrinol Metab 2007;92:1640-6. 
6. Leonard MB, Shults J, Wilson B, Tershakovec AM, Zemel BS. Obesity during childhood and adolescence augments bone mass and bone dimensions. Am J Clin Nutr 2004;80:51423.

7. Veldhuis JD, Roemmich JN, Richmond EJ, Bowers CY. Somatotropic and gonadotropic axes linkages in infancy, childhood, and puberty-adult transition. Endocr Rev 2006;27:101-40.

8. Prouteau S, Benhamou L, Courteix D. Relationships between serum leptin and bone markers during stable weight, weight reduction and weight regain in male and female judoists. Eur J Endocrinol 2006;154:389-95.

9. Tang ZH, Xiao P, Lei SF, Deng FY, Zhao LJ, Deng HY, et al. A bivariate whole-genome linkage scan suggests several shared genomic regions for obesity and osteoporosis. J Clin Endocrinol Metab 2007;92:2751-7.
10. Bonds DE, Larson JC, Schwartz AV, Strotmeyer ES, Robbins $\mathrm{J}$, Rodriguez $\mathrm{BL}$, et al. Risk of fractures in women with type 2 diabetes: the Women's Health Initiative Observational Study. J Clin Endocrinol Metab 2006;91:3404-10.

11. Hipponen E, Power C. Vitamin D status and glucose homeostasis in the 1958 British cohort. Diabetes Care 2006;29:2244-6.

Endereço para correspondência:

Francisco Bandeira

Rua da Hora 378

52020-010 Recife, PE 